\begin{tabular}{|c|} 
Continuing \\
medical \\
education
\end{tabular}

\section{How to assess an article on economic evaluation}

\author{
Mark Sculpher, Danielle Mercey
}

\section{Introduction}

CLINICAL SCENARIO

As a London based consultant in genitourinary medicine you are approached by your colleagues in the neighbouring obstetrics department who are concerned about the prevalence of HIV infection in their antenatal population, as determined by unlinked anonymous testing. As a result of the ACTG 076 study, ${ }^{1}$ it is your routine practice to offer zidovudine to all known HIV infected pregnant women, as this has been shown (a) to reduce the rate of vertical transmission, (b) to have no adverse maternal effects, and (c) to have no short term adverse effects for the neonate.

Your anonymous testing programme tells you how many HIV positive women deliver in the obstetrics unit each year, but it is apparent that the majority of these are not known to be positive. You have calculated the numbers of paediatric infections that could be prevented if (a) all the mothers accept screening, and (b) all positive mothers accept zidovudine treatment. However, before agreeing to implement this screening policy you are asked to provide evidence of the cost effectiveness of such a screening programme. Furthermore, you are asked to show how this cost effectiveness may alter with more effective means of reducing vertical transmission or if current antiretroviral therapies become less effective in reducing transmission as a result of the transmission of resistant viruses.

\section{Aim of this paper \\ The aim of this paper is to review a published economic evaluation of voluntary antenatal HIV screening in the United States undertaken by Mauskopf and colleagues to assess the strengths and weaknesses of the study and the implications of its results for patients. ${ }^{2}$ The structure of the review follows that of the McMaster series on critical appraisal of economic evaluations ${ }^{34}$ (see table 1 ). Readers interested in a general introduction to eco-}

nomic evaluation in health are directed towards other publications. ${ }^{5-7}$ The paper considered here uses decision analytic modelling as the framework within which to consider the costs and benefits of screening; a general introduction to these methods is also available elsewhere. ${ }^{89}$

\section{Summary of the economic results}

Mauskopf and colleagues' paper included two separate analyses. The first of these looked at the cost effectiveness of the use of zidovudine in reducing the rate of vertical transmission in women known to be HIV positive. The second analysis considered the cost effectiveness of a programme of voluntary screening of pregnant women for HIV and the treatment of positive cases with zidovudine. Given the clear need to identify women with HIV before the use of interventions to reduce the risk of vertical infection, it is this analysis that we focus on here. The net cost of a voluntary antenatal screening programme - that is, the cost of screening all women and administering zidovudine to positive cases net of the cost avoided of caring for HIV positive infants by a reduced vertical transmission rate-was found to be sensitive to a range of factors. At an acceptance rate for screening of $100 \%$, screening programmes would save money overall at all HIV prevalence rates between 5 and 50 per 1000 . The study found significant variation according to the assumptions used in the analysis.

\section{How valid were the results of the study?}

It is helpful to distinguish two general types of validity in economic evaluations: internal and external validity. Internal validity refers to the extent to which the estimates of cost and benefit have been generated using methods that are generally considered acceptable in the methodological literature. External validity refers to the extent to which the results are generalisable across a wide range of locations and clinical contexts. Much of the commentary below focuses on internal validity, but the applicability of the results to UK practice is a key issue for consideration which is also discussed.

WAS A FULL ECONOMIC COMPARISON UNDERTAKEN?

Were all the feasible options considered?

As for clinical evaluation using a controlled trial, a full economic evaluation requires a comparison of two or more interventions or programmes. ${ }^{5}$ In the study of Mauskopf et al, the comparison of interest was between a voluntary antenatal screening programme and 
no screening. Although this may represent the key clinical and economic comparison, additional options could have been considered. For example, the selection of the baseline option against which to compare a new screening programme should ideally be standard routine practice and, rather than no screening at all, it is likely that women would be tested on request. However, currently in the United Kingdom very few women are tested on this basis, ${ }^{10}$ so a change in the baseline comparator may not affect the conclusions markedly.

New screening options could feasibly be instituted as alternatives to universal voluntary screening and their cost effectiveness assessed. For example, although selective screening has been shown to produce variable and sometimes quite low uptake for antenatal hepatitis B screening in the United Kingdom, ${ }^{11}$ a selective programme for antenatal HIV testing, focusing on screening high risk women may, in some areas, be more cost effective. The potential cost effectiveness of selective screening was considered as a secondary analysis by Mauskopf et al rather than as an explicit option, and little detail was provided on the assumptions upon which this part of the analysis was based.

\section{Were both costs and benefits considered?}

Full economic evaluation requires the consideration of both the costs and outcomes of options. As explained above, the focus of the paper by Mauskopf et al was on the overall (net) costs of voluntary screening; in other words, the aim of the paper was to identify the conditions under which voluntary screening would be an economically dominant option. Dominance is said to exist when an option is both less costly and more effective than its comparator, and hence it is unequivocally cost effective. In this context, voluntary screening would dominate no screening if it has positive outcomes (that is, it reduces the rate of vertical HIV transmission) and also reduces costs.

However, variation in some of the assumptions and variable estimates was shown to increase costs overall and, in this context, an assessment of cost effectiveness requires the additional cost of the screening to be considered alongside its improved outcomes, usually in the form of a ratio of the incremental cost per additional unit of outcome. ${ }^{12}$ Mauskopf $e t$ al provided data on the incremental cost per case of HIV avoided in situations when screening is not dominant. However, in order for purchasers to compare the value in money of screening with other uses of health service resources in all areas of provision, it is necessary to estimate cost effectiveness ratios using generic measures of outcome that have meaning in other clinical areas. ${ }^{7}$ Mauskopf et al could, therefore, have presented the incremental costs per life year gained or per quality adjusted life year (QALY) gained when screening was not dominant to help resource allocation across programmes and specialties.

\section{Was a sufficiently broad costing perspective} adopted?

An important decision in all economic evaluations is what costing perspective to take-that is, costs to whom? In general, a continuum of perspectives is available. A narrow perspective would focus on a single budget such as pharmacy; some studies might broaden this to include all hospital costs. However, it is usually accepted that a cost analysis should at least cover all health service costs, and this is the focus of the paper by Mauskopf et al. Recent US guidelines for economic evaluation have argued that analyses should include a societal costing perspective, ${ }^{13}$ which would cover all resource costs no matter on whom they fell. As regards voluntary antenatal HIV screening, Mauskopf and colleagues' paper could have been strengthened by the inclusion of the costs to women of screening, in particular in terms of the time they commit to screening, and the costs of an infant with HIV/AIDS to social services. It is not clear whether broadening the costing perspective would alter the balance of the economic argument for voluntary screening.

WERE THE COSTS AND OUTCOMES PROPERLY MEASURED AND VALUED?

The paper by Mauskopf et al uses a decision analytical framework to synthesise data from a range of sources. Three general types of data were incorporated into the analysis-clinical effectiveness, epidemiological, and cost.

\section{Was clinical effectiveness established?}

The most important effectiveness variable was the reduction in the rate of vertical transmission as a result of administering zidovudine to pregnant women found to be HIV positive. This factor was taken from the AIDS Clinical Trials Group (ACTG) Protocol 076 which showed a vertical transmission rate of $8.3 \%$ with zidovudine compared with $25.5 \%$ without. ${ }^{1}$ This large American/French double blind, placebo controlled trial was terminated after a planned interim analysis showed a two thirds reduction in transmission in 363 infants for whom HIV status was known. The women were all well, had CD4 counts greater than 200, and most were antiretrovirally naive. Further placebo controlled trials would be unethical in this setting. Although the differences in transmission rate detected in the trial were statistically significant, there is uncertainty in the variable values incorporated into the economic analysis owing to statistical uncertainty within the trial and the fact that they are taken from only one study. Therefore, Mauskopf et al assess what the cost effectiveness of voluntary screening would be if zidovudine was less effective than shown in ACTG 076.

Several other aspects of effectiveness will influence the cost effectiveness of antenatal HIV screening but were not considered explicitly in Mauskopf and colleagues' paper. These include the contribution of breast feeding to reducing the risk of vertical transmission which is of considerable importance in London where the majority of HIV infected women who do 
Table 2 Derivation of the key costs in the model of Mauskopf et al

\begin{tabular}{|c|c|}
\hline Cost variable & Details of cost variable estimate \\
\hline Additional costs of treating the woman & $\begin{array}{l}\text { In the base case it was assumed that this would be zero apart from the use of zidovudine to reduce the risk of vertical } \\
\text { transmission. As part of the sensitivity analysis, the cost of } 1 \text { or } 2 \text { years of additional treatment for HIV was considered } \\
\text { based on treatment cost estimated in } 1993 \text { and updated for inflation }\end{array}$ \\
\hline Cost of paediatric HIV/AIDS & $\begin{array}{l}\text { In the base case analysis these costs were taken from a study using } 1991-2 \text { data updated for inflation, and assumptions } \\
\text { about the size of the proportions of infants with HIV who progress to AIDS early compared with late and the life } \\
\text { expectancy of these two groups (\$US82 } 708 \text { using a } 5 \% \text { annual discount rate). Higher costs were included in the } \\
\text { sensitivity analysis (up to } \$ 124416 \text { ) }\end{array}$ \\
\hline Screening costs & $\begin{array}{l}\text { The costs of the screening process included the cost of an initial ELISA test which was repeated once or twice for } \\
\text { positives which culminated in a confirmatory western blot test. The base case costs of these tests were } \$ 4.80 \text { (range } \\
\$ 2-\$ 65 \text { ) for the ELISA and } \$ 48.50 \text { (range } \$ 0-\$ 90 \text { ) for the western blot. Counselling costs included a pretest cost } \\
\text { (including offering the test) of } \$ 22 \text {, a post-test cost for negative tests of } \$ 33.20 \text {, and a post-test costs for positives of } \\
\$ 77.34\end{array}$ \\
\hline
\end{tabular}

not know their status breast feed, but when diagnosed opt to bottle feed. ${ }^{10}{ }^{14}$ It is estimated that the avoidance of breast feeding alone may reduce transmission by $14 \% .^{15}$

Another important area of effectiveness is the possible benefits of the new combination antiretroviral therapies that are now being used to treat both adults and infants with HIV, ${ }^{16}$ and that have largely developed since the paper by Mauskopf et al was submitted for publication. In the context of screening, early detection of both infected women and infants will allow the earlier use of these new therapies and, although there remains uncertainty about their effectiveness, this is expected to increase time until onset of AIDS and life expectancy.$^{17}$ Moreover, the use of these new treatments will increase the costs of caring for individuals with AIDS and may limit the opportunity for antenatal HIV screening to reduce overall costs because screening results in their earlier use. In this context, there is a need to quantify the health outcomes of screening to assess whether its costs are justified by its health benefits, but this was not undertaken by Mauskopf et al. However, technologies develop so quickly in the area of HIV/AIDS that it is difficult to reflect rapid changes in clinical practice in economic analysis.

Were epidemiological factors estimated properly? The key epidemiological factor in the economic evaluation, and one of the most important elements of the whole analysis, is the prevalence of HIV among pregnant women. Mauskopf et al use a estimate of 1.71 per 1000 in their base case (or primary) analysis, but describe the variation that exists between geographical areas and subgroups of women. The analysis addresses this variation by exploring the relation between the net costs of screening and prevalence. As well as uncertainty in this variable from the perspective of decision making in the United States, it is important to note that the prevalence rates used in the analysis may be quite inappropriate for decision making in other countries.

Another crucial factor in the analysis is the probability that women will accept antenatal HIV screening. The base case analysis assumed $100 \%$ acceptance, but assessed the effect on net costs of a rate as low as $75 \%$. Voluntary screening programmes are being initiated in London, but uptake rates to date have been less than $60 \% .{ }^{10}$ Furthermore, acceptance will be greatly influenced by hospital policy on how women are invited to take part in the programme. Given this uncertainty, it was surprising that the effect of an acceptance rate somewhat lower than $75 \%$ was not assessed, especially as the acceptance rate was found to be critical to the overall net cost of the programme.

\section{Were costs measured accurately?}

The three cost estimates that the analysis found to be most influential on the overall net cost of the screening programme were the additional costs of treating infected women as a result of earlier detection, the cost of caring for infants with HIV/AIDS, and the cost of screening. Table 2 details the sources of and assumptions behind each of these cost estimates. Given their importance to the overall results of the analysis, it is correct to subject these particular cost estimates to detailed scrutiny. Both the additional cost of treating HIV positive women and the cost of paediatric HIV/AIDS were taken from earlier studies based on data collected before the advent of combination therapies. Although the effects of higher values for these cost variables were assessed, it is likely that these costs estimates are out of date and this may threaten the validity of the results. The screening costs too may not accurately reflect those that would exist if voluntary screening were made routine practice. Assumptions that had been made about the time taken to explain the test to women, for example, were not fully detailed in the paper.

Were costs and outcomes appropriately integrated? In order to assess the cost effectiveness of an intervention or programme it is necessary to relate the costs to the outcomes. As discussed above, under most assumptions the screening programme was found to save money overall; given that it also facilitates a reduction in HIV cases, the programme is said to be a dominant one and unequivocally cost effective. Under some assumptions, however, screening was found to increase costs overall. Under these circumstances, decision makers would benefit from being informed of the incremental cost per unit of health gain as a result of screening; but this information was not provided.

Was appropriate allowance made for uncertainty in the analysis?

In all economic evaluations uncertainty is unavoidable in areas relating to data inputs, 
methodological decisions, and the generalisability of the results. ${ }^{18}$ Economic evaluations of antenatal HIV screening programmes are particularly prone to uncertainty given that they include a number of clinical, epidemiological, and cost elements over a long term time horizon. Sensitivity analysis is used widely in economic evaluation to assess how robust the results of an analysis are to changes in variable estimates. ${ }^{18}{ }^{19}$ It involves altering one or more variables in the analysis within a plausible range (for example, the cost of an HIV test) and observing how this affects the overall estimates of cost and/or effectiveness. The less sensitive the results to changes in variables, the more robust the analysis. Mauskopf et al use sensitivity analysis extensively and their focus is to explore whether changes in variables are sufficient to cause the screening programme to have positive net costs overall. They found that the results of the analysis were most sensitive to the prevalence of HIV in pregnant women, the acceptance rate of voluntary screening, the cost of paediatric HIV, the transmission rate following use of zidovudine, screening costs, women's willingness to be treated with zidovudine, and the additional cost of caring for HIV positive women as a result of screening. Given this multidimensional uncertainty, it is important to consider whether the sensitivity analysis considers the full range of values for uncertain variables. For example, the effect of high screening costs based on high costs for the screening test and counselling was not fully assessed in the sensitivity analysis. Full details of sensitivity analyses are often difficult to provide given constraints on space in journals, so it is an advantage of studies if authors are willing to share further analyses with interested readers or even to provide the computer model itself for decision makers to incorporate their own variable values. It may be the case that a fuller sensitivity analysis would indicate that there are rather more combinations of variable values that would result in positive costs overall from screening, which would emphasise the need to indicate the level of health gain resulting from the incremental costs.

Are estimates of costs and outcomes related to the baseline risk in the treatment population?

It is important to establish whether an economic evaluation has reported the extent to which costs, outcomes, and overall cost effectiveness are related to the characteristics of the patient population. For example, in a study of the cost effectiveness of streptokinase compared with tissue plasminogen activator (t-PA) as thrombolytic therapies for acute myocardial infarction, the cost per life year gained of the more costly therapy (t-PA) was strongly related to the age of the patient and the location of the infarct. ${ }^{20}$ In the context of voluntary antenatal HIV screening, which focuses on populations rather than specific patient groups, the key measure of baseline risk is the prevalence of HIV in pregnant women, and Mauskopf and colleagues' paper clearly indicates the strong relation between the net overall cost of the screening programme and prevalence. As argued above, the importance of this factor suggests that selective screening programmes focused on high risk groups may be more cost effective than voluntary screening, at least in some areas.

\section{Will the results answer the questions in the introductory clinical scenario?}

This paper compared a voluntary screening programme with no testing. Although a few hospitals in the United Kingdom have established screening programmes, none has achieved an uptake of $75 \%,{ }^{10}$ this paper's worst case estimate. This limits the usefulness of the comparison.

Mauskopf et al used a range of HIV prevalences in pregnant women in their model and predicted cost savings if the prevalence were between 5 and 50 per 1000 . In the United Kingdom, the prevalence of HIV, as determined by anonymous testing of child bearing women in 1996 , was $0.19 \%$ in London but only $0.016 \%$ elsewhere in the United Kingdom, excluding Scotland. ${ }^{21}$ This does not mean that universal screening should not be considered outside London, but reinforces the need for estimates of the incremental cost per unit of health gain in evaluating overall cost effectiveness of such programmes.

In ACTG 076 the transmission rate of $25.5 \%$ in the untreated arm was higher than that observed in some European studies and this could mean that the cost savings in Europe would be less. However, concerns that a vertical transmission rate of only $8.3 \%$ might not be achieved outside the trial situation seemed to have been unfounded and where zidovudine is widely used transmission rates of around 5\% have been observed. ${ }^{22}$ There remains concern, however, that as more women develop resistant virus, either through prior therapy (not applicable in this scenario as we are considering screening women not known to be positive) or through transmission of resistant virus, the efficacy of a single antiretroviral agent in reducing vertical transmission may fall.

Mauskopf et al assumed that all diagnosed women would agree to treatment, in their base case scenario, but considered refusal rates of up to $50 \%$. Gibb et al have found acceptance rates of antiretroviral therapy among women aware of their HIV positive status of $75 \%$ in the United Kingdom. ${ }^{14}$

\section{Conclusion}

Mauskopf et al show that many different factors will determine whether or not universal screening of pregnant women will offer cost savings. Many of the variable ranges used include those likely to be found in parts, at least, of the United Kingdom. The data presented in the analysis will augment the results of a UK based cost effectiveness analysis funded by the Department of Health which is due to report in 1998 .

1 Connor E, Sperling R, Gelber R, et al. Reduction of maternal-infant transmission of human immunodeficiency virus type 1 with zidovudine treatment. $N$ Engl f Med 1994;33:173-80. 
2 Mauskopf JA, Paul JE, Wichman DS, et al. Economic impact of treatment of HIV-positive pregnant women and their newborns with zidovudine. $\mathcal{F} A M A$ 1996;276:132-9.

3 Drummond MF, Richardson S, O'Brien BJ, et al. Users' guide to the medical literature. XIII. How to use an article on economic analysis of clinical practice. A. Are the results of the study valid? $\mathcal{F} A M A 1997 ; 277: 1552-7$.

4 O'Brien BJ, Heyland D, Richardson S, et al. Users' guide to the medical literature. XIII. How to use an article on economic analysis of clinical practice. $B$. What are the results and will they help me in caring for my patients? $f A M A$ 1997;277:1802-6

5 Drummond MF, O'Brien BJ, Stoddart GL, et al. Methods for the economic evaluation of health care programmes. New York: Oxford University Press, 1997.

6 Sloan F, ed. Valuing health care: costs, benefits and effectiveness of pharmaceuticals and other medical technologies. Cambridge:

7 Sculpher MJ. Economic evaluation in obstetrics and gynaecology. Baillière's Clin Obstet Gynaecol 1996;10:661-76.

8 Weinstein MC, Fineberg HV. Clinical decision analysis. Philadelphia: WB Saunders, 1980.

9 Sox HC, Blatt MA, Higgins MC, et al. Medical decision making. Stoneham, MA: Butterworths, 1988.

10 Gibb DM, MacDonagh SE, Gupta R, et al. Factors affecting the uptake of antenatal HIV testing in London: results of a multicentre study. BMF 1998;316:259-61.

11 Boxall E. Antenatal screening for carriers of hepatitis B virus. $B M F$ 1 1995;311:1178-9.

12 Doubilet P, Weinstein MC, McNeil BJ. Use and misuse of the term 'cost effective' in medicine. $N$ Engl $f$ Med the term 'cost
13 Gold MR, Siegel JE, Russell LB, et al. Cost-effectiveness analysis in health and medicine. New York: Oxford University Press, 1996.

14 Gibb DM, MacDonagh SE, Tookey PA, et al. Uptake of interventions to reduce mother-to-child transmission of HIV in UK and Eire. AIDS 1997;11:F53-8

15 Dunn DT, Newell, L, Ades AE, et al. Risk of human immunodeficiency virus type 1 transmission through breastfeeding. Lancet 1992;340:585-8.

16 British HIV Association Guidelines Coordinating Committee. British HIV Association guidelines for antiretroviral treatment of HIV seropositive individuals. Lancet 1997;349:1086-92.

17 Deeks S, Smith M, Holodniy M, et al. HIV-1 protease inhibitors. FAMA 1997;277:145-53.

18 Briggs A, Sculpher M, Buxton $M$. Uncertainty in the Briggs A, Sculpher $M$, Buxton $M$. Uncertainty in the
economic evaluation of health care technologies: the role of sensitivity analysis. Health Econ 1994;3:95-104

19 Briggs A, Sculpher M. Sensitivity analysis in economic evaluation: a review of published studies. Health Econ $1995 ; 4: 355-71$

20 Mark DB, Hlatky MA, Califf RM, et al. Cost effectiveness of thrombolytic therapy with tissue plasminogen activator as compared with streptokinase for acute myocardial infarction. N Engl F Med 1995;332:1418-24.

21 Nicol A, McGarrigle C, Brady A, et al. Epidemiology and detection of HIV-1 among pregnant women in the United Kingdom: results from national surveillance 1988-96. KMF 1998;316:253-8.

22 Fiscus S, Adimora A, Schoenbach V. Perinatal HIV infection and the effect of zidovudine therapy on transmission in rural and urban counties. $¥ A M A$ 1996;275:1483-8. 